\title{
Distribution of Aortic Mechanical Prosthetic Valve Closure Sound Model Parameters on the Surface of the Chest
}

\author{
Ahmet Baykal, Y. Ziya İder, and Hayrettin Köymen, Senior Member, IEEE
}

\begin{abstract}
It has been previously proposed that heart valve closure sounds can be modeled by a sum of decaying sinusoids, based on the hypothesis that the heart cavity, heart walls, major vessels, and other structures in the chest constitute a frequency selective linear acoustic system and this system is excited by the rapidly decelerating valve occluder. In this study, distribution of the parameters of this model for the second heart sound is investigated. For this purpose, heart sounds of 10 patients who have a St. Jude-type bileaflet mechanical heart valve prosthesis in aortic position are recorded. Recordings are performed at $\mathbf{1 2}$ different locations on the surface of the chest. To reliably assign representative parameters to each recording site, signal averaging, model order selection, and a special filtration technique are employed. The results of the analyses are discussed in relation to the above hypothesis on the heart sound generation mechanism. It is observed that site-to-site variation of frequencies of modes does not exceed the accuracy limit of proposed analysis method, but energies of these modes vary on the surface of the chest, and as a result of statistical analysis, it appears that energy of some modes are significantly different between two recording sites.
\end{abstract}

\section{INTRODUCTION}

$\mathbf{S}$ PECTRAL analysis of the heart sound has been used by researchers for different purposes. Several investigators have reported that by using such a technique, anatomical or physiological malfunctions in heart valve prostheses can be differentiated [1]-[6]. It is also reported that power spectrum of the heart sound may vary in relation to certain cardiovascular disorders [7]-[11].

In all of those studies, different spectral parameters have been used. Gordon et al. used maximum frequency as a diagnostic criterion and they observed that mechanical malfunction of a heart valve prosthesis causes a decrease in this parameter [2]. Kagawa et al. have reported that the normalized maximum frequency (upper cut-off frequency 30 $\mathrm{dB}$ below the maximum) of power spectrum decreases during the postoperative course of patients with thrombosed valves [3]. Stein et al. studied patients with degenerated porcine bioprosthetic valves. They defined "dominant frequency" as

Manuscript received August 19, 1991; revised February 19, 1993 and December 19, 1994

A. Baykal and Y. Z. İder are with the Department of Electrical and Electronics Engineering, Middle East Technical University, 06531 Ankara, Turkey.

H. Köymen is with the Electrical and Electronics Engineering Department, Bilkent University, 06530 Ankara, Turkey.

IEEE Log Number 9409107. the frequency of the highest magnitude shown in the sound spectrum. They reported that this parameter is higher in patients with degenerated heart valve prosthesis than that in normal subjects [5]. In a recent study, Durand et al. extracted 18 diagnostic features from the sound spectrum and they used these parameters to distinguish subjects with normal valves from those with degenerated valves [6].

In most of these studies, investigations have been based on some heuristic features derived from the power spectrum. Variations of these heuristic features have been analyzed between subjects with normal valves and those with degenerated valves. These features are not related to the parameters of a particular model which attempts to explain the heart sound generation mechanism. Therefore, it is difficult to use these features for a better understanding of heart sound generation mechanism. If, however, parameters of a model which has been built by considering the influence of anatomical and physiological factors on heart sound generation were used, then better understanding of heart sound generation and better diagnosis of pathological situations would be achieved. Especially in follow-up studies, model-based approaches can be more sensitively used to follow the development of pathology [12].

Several different views about sound generation mechanisms have been adopted by different investigators. Sikarskie $e t$ al. proposed a mathematical model for aortic valve vibration and its relation to the second heart sound [13]. But they did not undertake a study to verify this model using PCG signals. Furthermore, this model only considered a valvular origin for heart sound, whereas other investigators emphasize the contribution of the oscillating structures in the chest [7], [10], [14]. However, they did not formulate mathematical models to explain their assumptions. As an extension to the emphasis put by these investigators, it was assumed in a recent study that heart sounds are generated as a response of a frequency-selective linear-acoustic system to the rapidly decelerating heart valve occluder [12]. It is considered that this system consists of the entire heart cavity, heart walls, major vessels, and other structures in the chest, all having natural resonance modes. In line with this hypothesis, heart sounds are expressed as a summation of damping sinusoids. Although a detailed correspondence between the parameters of this model and anatomical and physiological factors was not established, it was nevertheless shown that by estimating the parameters using Prony estimation technique [15], a good fit 
was obtained between the model parameters and actual heart sound signal.

In order to verify a model, it is necessary to test on a quantitative basis the implications of the hypotheses which stem from the specific assumptions about the heart sound generation mechanisms themselves. For example, if the resonant structure assumption is true, then frequencies of these resonance modes will not vary from one recording site to another on the surface of the chest, but the energy content of these modes will be different depending on the distances of the recording sites to the anatomical structures having natural resonance modes. It must be emphasized that such a hypothesis can not be tested using the above-mentioned heuristic features of the power spectrum of the heart sound. For example, frequency of peak amplitude of the power spectrum may change due to a change in the energies of the contributing resonance modes and not due to a change in their frequencies.

The purpose of this study is to investigate the variation of the modal parameters of second heart sounds on the chest surface and to identify whether any variations are due to frequency shifts or energy variations of the individual damped sinusoids on different recording sites. Closure sounds originating from metallic prosthetic valves are particularly suitable for such an investigation since the rapid deceleration of the valve occluder provides excitation, which has a wide frequency spectrum, to the acoustic system, comprising heart, major vessels, and other structures in the chest [12]. Furthermore, the aortic component in this sound is very high compared to the pulmonary one, hence the interference effect of the latter is considerably less.

In this study, heart sounds are recorded from 10 patients who have a St. Jude-type bileaflet mechanical heart valve prosthesis in aortic position. Heart sounds are recorded at 12 different sites on the surface of the chest. Second heart sounds are selected and analyzed. Estimated parameters for each site are compared. Such a comparison requires a precise method for parameter identification. For this purpose, an algorithm is developed which aims at reliably assigning representative parameters to a given specific recording site, despite noise and some physiological variation. Signal averaging is employed and a model order selection procedure and special filtration technique are proposed. The results of the analyses are discussed.

\section{METHODS}

\section{A. Recording}

Heart sounds of 10 patients who have St. Jude-type bileaflet mechanical heart valve prostheses implanted in aortic position, are recorded and second heart sounds are selected for analysis. Recordings are made by a Nihon Kohden (model RS5) PCG amplifier with $-3 \mathrm{~dB}$ cutoff frequencies measured to be at 100 and $1940 \mathrm{~Hz}$, and with $18 \mathrm{~dB} /$ octave fall-off outside the pass-band. The PCG transducer is of piezoelectric type (air coupled, Electronics for Medicine Inc., No. 03040502) and has a flat response in the range of frequencies mentioned above. Electrocardiograms are simultaneously recorded to be able to locate the second heart sound which occurs after the $T$ wave.
The outputs of the PCG and ECG amplifiers are recorded by a multichannel FM tape recorder which has a $0-5 \mathrm{kHz}$ flat frequency response. At playback, PCG signals are lowpass filtered (-48 dB/octave) at $500 \mathrm{~Hz}$ to prevent frequency aliasing. PCG and ECG are subsequently sampled and digitized by a 12-b A/D converter at a rate of $2 \mathrm{kHz}$ and stored on diskettes. An IBM PC/AT personal computer is used to control data acquisition procedures and for off-line analysis. Operator interactive signal monitoring software displays the recorded PCG and ECG for a few cardiac cycles. One of the second heart sounds is selected as a template and the other second heart sounds are detected and aligned using cross-correlation for signal averaging purposes. In each recording site, 2-9-s heart sounds, which have more than $90 \%$ correlation with the reference template, are selected and then averaged. Averaged second heart sounds are then analyzed.

\section{B. Parameter Identification}

In this study, second heart sounds are modeled as the sum of decaying sinusoids (modes). In this model, if $x_{n}$ represents the heart sound sampled at the $n$th sampling instant, then $x_{n}$ is expressed as

$$
\begin{aligned}
x_{n} & =\sum_{k=1}^{K} A_{k} \exp \left(-\alpha_{k} n T\right) \operatorname{Cos}\left(2 \pi f_{k} n T+\Theta_{k}\right)+e_{n} \\
n & =1 \ldots . N
\end{aligned}
$$

where

$N$ is the number of samples,

$K$ is the number of modes,

$A_{k}$ is the amplitude of the $k$ th mode,

$\alpha_{k}$ is the damping constant of the $k$ th mode,

$f_{k}$ is the frequency of the $k$ th mode,

$\Theta_{k}$ is the phase of the $k$ th mode,

$T$ is the sampling interval,

$e_{n}$ is the noise at the $n$th time instant.

The amplitude, damping constant, frequency, and phase of each mode are estimated by recursive implementation of the Prony method. SER $_{K}$ which is defined as

$$
\mathrm{SER}_{K}=10 \log \left(\left(\sum_{n=1}^{N} x_{n}^{* 2}\right) /\left(\sum_{n=1}^{N} e_{n}^{* 2}\right)\right)
$$

is the signal-to-error ratio (in $\mathrm{dB}$ ) for a model having $K$ modes, where

$x_{n}^{*}$ is the $n$th samples of estimated signal,

$e_{n}^{*}$ is the difference at $n$th sampling instant, between actual measurement and the prediction of the model (also called residual error).

Once the parameters of a model are identified, then the energy of the $k$ th mode, $E_{k}$ is calculated, using

$$
\begin{aligned}
E_{k} & =\int_{0}^{\infty} A_{k}^{2} \exp \left(-2 \alpha_{k} t\right) \operatorname{Cos}^{2}\left(2 \pi f_{k} t+\Theta_{k}\right) d t \\
& =\frac{A_{k}^{2}\left(\alpha_{k}^{2}+\left(2 \pi f_{k}\right)^{2}+\alpha_{k}^{2} \operatorname{Cos} 2 \Theta_{k}-2 \pi f_{k} \alpha_{k} \operatorname{Sin} 2 \Theta_{k}\right.}{4 \alpha_{k}\left(\alpha_{k}^{2}+\left(2 \pi f_{k}\right)^{2}\right)} .
\end{aligned}
$$


TABLE I

Parameters of the Artificial Closure Sound

\begin{tabular}{rrrcc} 
fre & damp & ampl & phase & energy \\
\hline 120 & 90 & 1000.0 & 4 & 1.000 \\
170 & 100 & 985.4 & 0 & 1.000 \\
220 & 150 & 1169.9 & 2 & 1.000
\end{tabular}

TABLE II

Estimated Parameters of the Noiseless Simulated Signal

\begin{tabular}{rrrrc} 
fre & damp & \multicolumn{1}{c}{ ampl } & phase & energy \\
\hline 120 & 90.1 & 1001.3 & 3.999 & 1.000 \\
170 & 100.3 & 987.9 & 0.000 & 1.000 \\
220 & 149.9 & 1170.1 & 2.001 & 0.999
\end{tabular}

1) Determination of the Model Order: Determination of the number of modes is of critical importance. We have done simulations to study the interrelations between mode number, parameter estimates, and the Signal-to-Noise ratio. A signal with three modes, the parameters of which are given in Table I, is generated and random sequences (zero mean, Gaussian sequence) of different variances are added to this signal. Thus, artificial closure sounds with SNR's of 21, 19, $15,11,8,6,4$, and $0.5 \mathrm{~dB}$ are obtained. Here, SNR is defined as

$$
\mathrm{SNR}=10 \log \left(\left(\sum_{n=1}^{N} x_{n}^{2}\right) /\left(\sum_{n=1}^{N} e_{n}^{2}\right)\right)
$$

where $e_{n}$ is the random noise at the $n$th time instant.

All signals are analyzed at $K$ of $3,6,10,15$, and 20 . It is observed that if the signal is noiseless, correct parameters can be estimated at a $K$ value of three (Table II). However, if the signal is noisy, accurate estimates $(<2 \%$ error for frequencies, $<10 \%$ error for relative energies of modes) can not be achieved when the model order is less then 10 .

When Akaike Information Criterion (AIC) [16], which is a widely used method for order selection in AR models, is used in the AR modeling step of Prony technique to determine the model order, an order of five was indicated for all SNR used. This estimate is, of course, close to the number of poles in the AR model of a signal containing three decaying sinusoids. However, from the perspective of obtaining accurate estimates of parameters, the order determined by AIC was not sufficient.

In Fig. 1, $\mathrm{SER}_{K}$ of artificial closure sounds are shown. In this figure, the orders for which accurate estimates can be achieved are marked by " $\boldsymbol{\square}$." For these orders, estimation errors are between $0.1 \%$ and $2 \%$ for frequencies and $0 \%$ and $10 \%$ for relative energies. It can be seen from Fig. 1 that, as the model order is increased, $\mathrm{SER}_{K}$ increases. For a low noise case ( $S N R=21 \mathrm{~dB}$ ), the SER $_{K}$ curve shows a sharp corner and reaches a plateau. This corner occurs at $K=10$ and accurate frequency and energy estimation $(<2 \%$ error for frequencies, $<10 \%$ error for relative energies of modes) can be achieved for this and higher orders. For the signal for which SNR is $19 \mathrm{~dB}$, the corner in the SER $K$ curve becomes smoother and accurate estimates can be achieved for $K$ 's of 15 and 20. In other cases for which SNR's are less than 19,

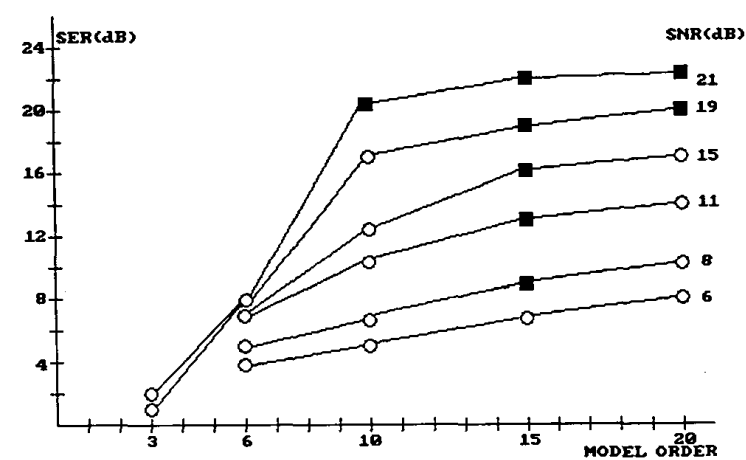

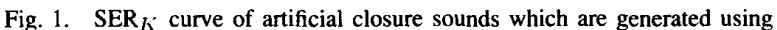
the parameters given in Table I. The orders at which accurate estimates can be achieved are marked by " $\square$ " symbols. Accurate estimates can not be achieved at the orders marked by "O" symbols.

such a corner can not be observed. For the cases for which SNR's are between 19 and $6 \mathrm{~dB}$, accurate estimates can only be achieved at $K$ of 15 . In the last case (SNR $=6 \mathrm{~dB})$, no acceptable estimates can be found.

These observations imply that, when the noise level of the signal is sufficiently low, the SER $_{K}$ curve shows a sharp corner. For the $K$ value at which this corner occurs, accurate estimates can be achieved. If the noise level of the signals is relatively high, a corner in the $\mathrm{SER}_{K}$ curve can not be identified and, in these cases, using a high value of $K$ does not necessarily yield correct parameter estimates. Indeed, if the model order is increased, $\mathrm{SER}_{K}$ increases, but the estimates become erroneous. Therefore, in the analysis of actual heart sounds, reduction of the noise level is of critical importance. For this purpose, signal averaging is found helpful.

For averaged heart sounds, the mode number is determined as follows: Signals are analyzed for increasing model orders, starting at $K=6$. In each successive step, $K$ is increased by two. As $K$ is increased, SER $_{K}$ increases and reaches a plateau. This curve may show a sharp corner depending on the level of noise. $K$ for this corner is accepted as the model order. If such a corner can not be identified, the analysis is deemed invalid.

2) Filtration of Lower Frequency Modes: The principal aim of this study is to understand how the energies and frequencies of modes comprising a heart sound vary on the surface of the chest. The modeling and estimation techniques employed to this end must incorporate all precautions against the factors which reduce the precision of results. Apart from the effects of noise which are discussed in the previous section, signal components which can not be recorded with high fidelity are a serious concern.

Heart sound analyses used for clinical purposes are usually made in $100-200 \mathrm{~Hz}$ (for mid-frequencies) or $200-500 \mathrm{~Hz}$ (for high frequencies) [17]. For power spectrum analysis, different investigators have used different cut-off frequencies. For example, Arnott et al. used a band-pass filter with cut-off frequencies of 20 and $1500 \mathrm{~Hz}$ [10], on the other hand, Stein et al. recorded sound signals between $50-500 \mathrm{~Hz}$ [5]. Our PCG recording system has a high-pass filter with a $-3 \mathrm{~dB}$ frequency of $100 \mathrm{~Hz}$. However, most of the energy in the filtered signal 


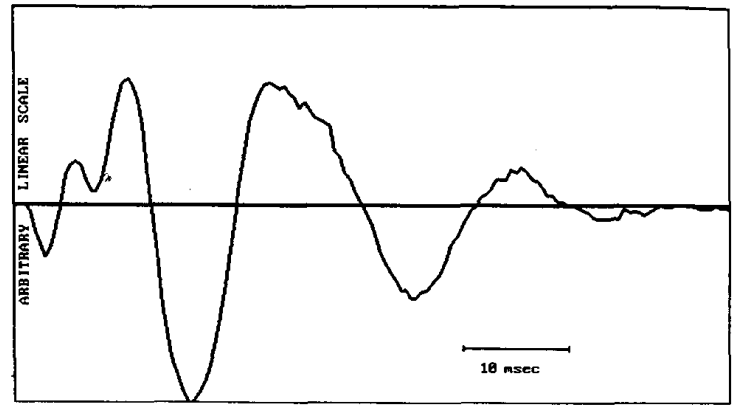

(a)

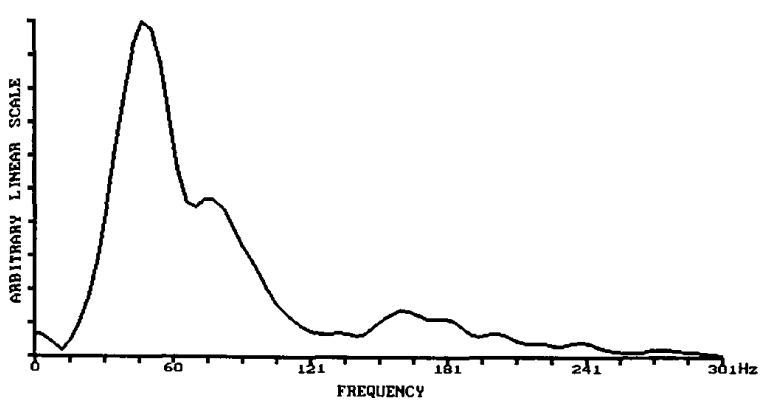

(b)

Fig. 2. (a) A typical closure sound. (b) DFT of this signal.

TABLE III

Parameters of the Closure Sound Given in Fig. 3. Energies ARE Normalized to the Energies of the Modes at $99 \mathrm{~Hz}$

\begin{tabular}{rrrcr}
\multicolumn{1}{c}{ fre } & \multicolumn{1}{c}{ damp } & \multicolumn{1}{c}{ ampl } & phase & energy \\
\hline 37.7 & 97.1 & 9231.7 & 0.394 & 91.491 \\
47.1 & 177.9 & 13283.7 & 3.864 & 72.184 \\
55.1 & 94.4 & 6902.7 & 1.605 & 58.516 \\
75.2 & 77.2 & 1296.8 & 1.844 & 2.824 \\
99.3 & 103.5 & 1004.6 & 4.097 & 1.000 \\
171.7 & 111.8 & 411.5 & 0.002 & 0.287 \\
284.9 & 348.0 & 263.6 & 1.893 & 0.026
\end{tabular}

still lies below $100 \mathrm{~Hz}$ (Fig. 2). These components, on the other hand, do not lend themselves to a precise modal analysis because they are critically effected by the filter. In particular, minor variations in the modal frequency result in significant changes in modal energy if this signal component lies in the rising edge of the high-pass filter. Therefore, it is not appropriate to include these modes in our comparative study. Furthermore, the presence of these modes causes inaccuracies in the estimation of parameters of the components which are in the pass-band. Simulation studies were undertaken to demonstrate these effects.

For simulation purposes, an artificial closure sound is generated using (1) with $K=7$ and the parameters shown in Table III which correspond to signals depicted in Fig. 2(a). $e_{n}$ is selected as a zero-mean Gaussian sequence such that the SNR for this artificial signal is $22.5 \mathrm{~dB}$.

The artificial signal is shown in Fig. 3. The Prony method is first applied to this signal without any prior filtering. The mode numbers are taken as 10,12,14,16,20,22, and 24 .

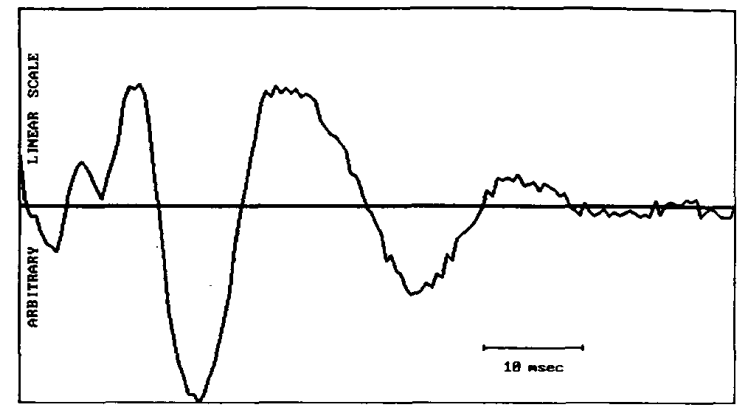

Fig. 3. Artificial closure sound which is generated using the parameter given in Table III.

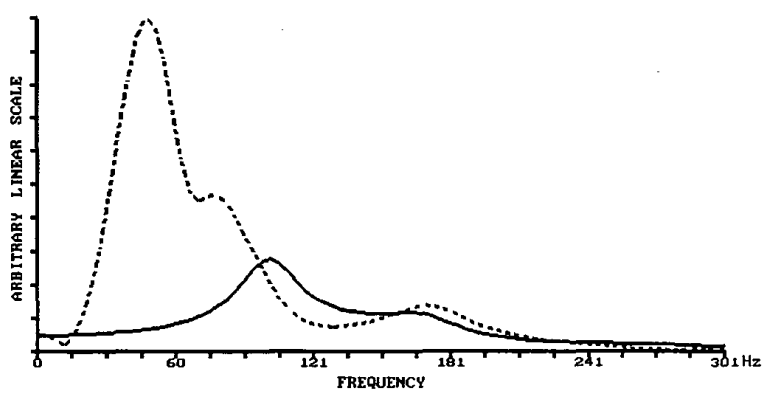

Fig. 4. DFT's of artificial closure sound, before (dashed line) and after (solid line) modal high-pass filtration.

The frequencies and energy of high-frequency modes can not be accurately estimated (error $<2 \%$ in frequency and $<10 \%$ in energy) in any of these cases. In fact, obtained errors are not even below $5 \%$ for frequencies and $25 \%$ for energies.

In this study, a special "modal high-pass filtering technique" is proposed in order to reduce the effect of low-frequency modes. The parameters of these modes to be minimized are estimated first in this technique. To do this correctly, the frequency band of the signal must be as small as possible for accurate estimation. On the other hand, it must be large enough to cover most of the energy contained in significant low frequency modes. We chose this frequency as $140 \mathrm{~Hz}$ based on our observations regarding the range of the damping constants of low-frequency modes.

This technique is implemented on the simulated signal. The DFT of the artificial closure sound is obtained (dashed line in Fig. 4), components of the complex DFT above $140 \mathrm{~Hz}$ are zeroed, and an inverse DFT is obtained. When the Prony method is applied to this signal, an SER of $40 \mathrm{~dB}$ is obtained. A signal is then reconstructed using the estimated modes with center frequencies below $90 \mathrm{~Hz}$ and this signal is subtracted from the original artificial signal. The DFT of this signal is given in Fig. 4 (solid line). We refer to this new artificial signal as the modal high-pass filtered signal. In Fig. 5, this filtration technique is shown in a block diagram. When the Prony method is applied to the modal high-pass filtered signal, the frequencies and energies of the modes above $90 \mathrm{~Hz}$ are found with an accuracy of $<2 \%$ for frequencies and $<16 \%$ for energies (Table IV). An SER of $14 \mathrm{~dB}$ is obtained in this case. 


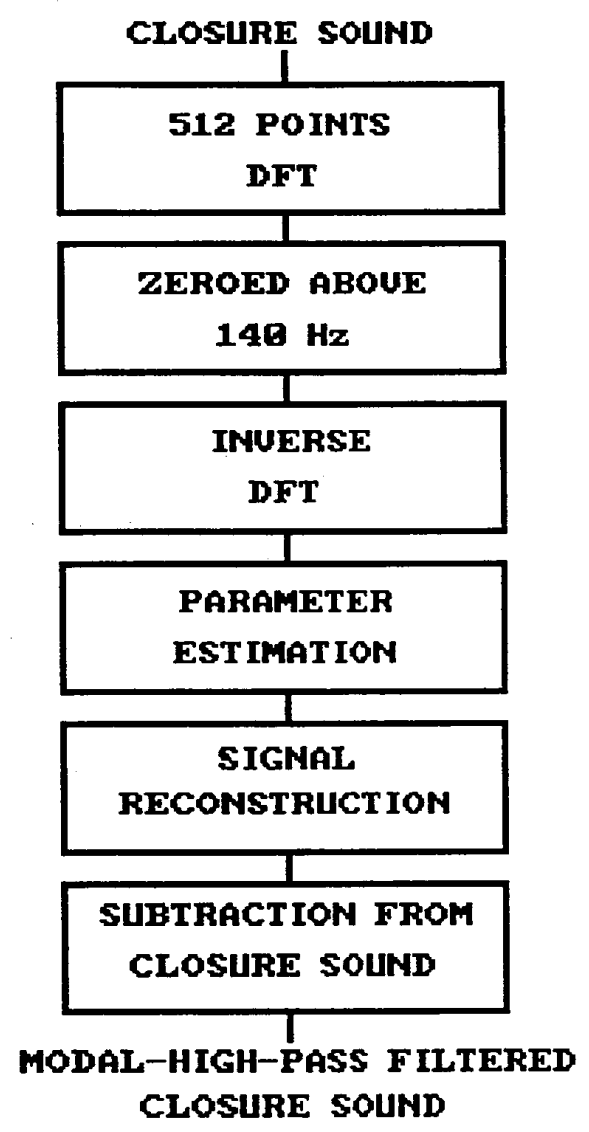

Fig. 5. Block diagram of modal high-pass filtering.

The error levels obtained in this highly idealized simulation are difficult to attain in the study of actual signals. First of all, such a high $\operatorname{SER}_{K}(40 \mathrm{~dB})$ is not always possible in the estimation of low-frequency components. Furthermore, model order determination is still prone to errors, hence additional variation in parameter estimates is expected. When the noise level is increased so that the SNR is decreased to $21 \mathrm{~dB}$ from $22.5 \mathrm{~dB}$, it is observed that the three high-frequency modes can still be estimated with errors less than $4.2 \%$ in frequency and $26 \%$ in energy, while a reliable order determination can still be done at $12.4 \mathrm{~dB} \mathrm{SER}_{K}$. If the SNR is further decreased to $20 \mathrm{~dB}$, the maximum error in frequency and energy becomes $5.2 \%$ and $28 \%$, respectively, but choosing the correct order according to the above procedure is not possible. Obviously, these figures are dependent on the number of modes included, on the relative energy of each mode, and on the noise level. For comparison purposes, $5 \%$ estimation error for frequencies and $25 \%$ error for energies are taken as the limitations of the whole identification procedure.

The modal high-pass filtering technique can be summarized as follows:

1) The DFT of the heart sound is calculated.

2) The DFT is zeroed above $140 \mathrm{~Hz}$.

3) The inverse DFT is applied.

4) The Prony method is used to estimate the parameters of this low-pass filtered signal.
TABLE IV

Estimated Parameters of the Modal High-Pass Filtered artificial. Closure Sound. Only the Parameters of the Modes Which Have More than 1\% Normalized Energies ARE Given

\begin{tabular}{ccccc} 
fre & damp & ampl & phase & energy \\
\hdashline-100.2 & 80.2 & 605.9 & 4.271 & 1.000 \\
169.8 & 115.8 & 458.4 & 0.068 & 0.443 \\
288.3 & 241.9 & 120.3 & 1.218 & 0.013
\end{tabular}

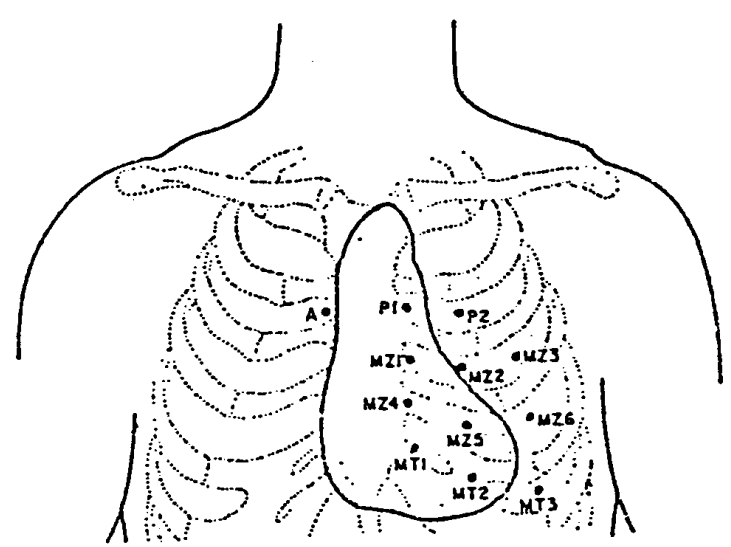

Fig. 6. Twelve recording sites on the surface of the chest.

5) A signal is reconstructed using the parameters of modes with less than $90 \mathrm{~Hz}$ center frequency.

6) This constructed signal is subtracted from the original signal to obtain the modal high-pass filtered signal.

\section{RESULTS}

\section{A. Data}

Recordings are examined from 10 patients who have a St. Jude-type bileaflet mechanical heart valve prosthesis in aortic position. Ages of the patients are between 18 and 40 . Patients 3 and 9 are female and the others are male. Diameters of the prostheses are $21-27 \mathrm{~mm}$. Recordings are made two or six months following the implantation of the prosthesis.

\section{B. Site-to-Site Variation of the Second Heart Sounds}

To observe the site-to-site parameter variation of the second heart sounds, 12 recording sites are selected on the surface of the chest. These recording sites are illustrated in Fig. 6. The anatomical definitions of these points are as follows:

A: Intersection point of second intercostal space with right sternal border.

P1: Intersection point of second intercostal space with left sternal border.

P2: In the second intercostal space, mid point between P1 and intersection point of second intercostal space with left medio-clavicular line.

MZ1: Intersection point of third intercostal space with left sternal border.

MZ2: In the third intercostal space, mid point between MZ1 and MZ3. 

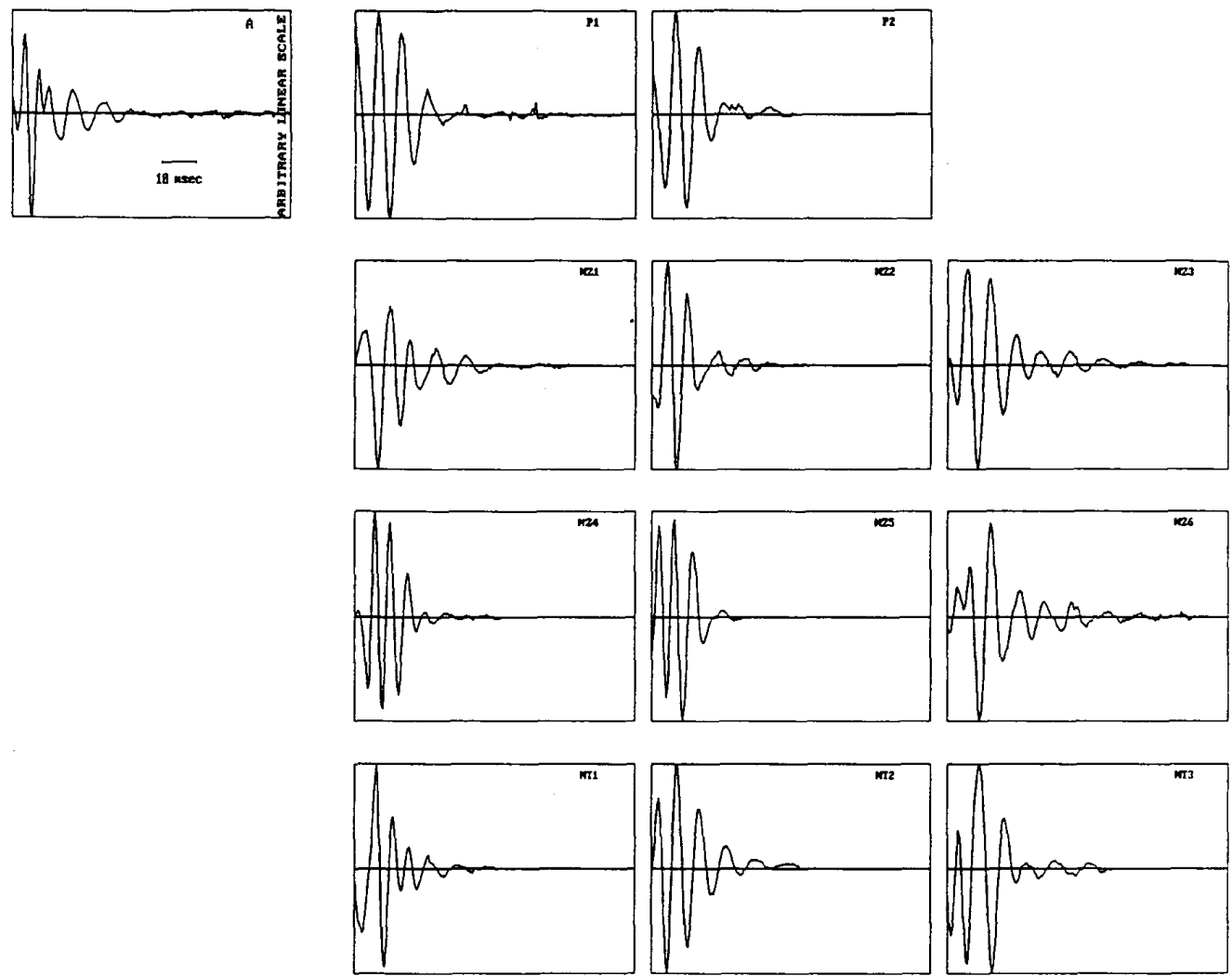

Fig. 7. Averaged closure sounds recorded at 12 different sites.

MZ3: Intersection point of the third intercostal space with the left medio-clavicular line.

MZ4: Intersection point of fourth intercostal space with left sternal border.

MZ5: In the fourth intercostal space, mid point between MZ4 and MZ6.

MZ6: Intersection point of the fourth intercostal space with the left medio-clavicular line.

MT1: Intersection point of fifth intercostal space with left sternal border.

MT2: In the fifth intercostal space, mid point between MT1 and MT3.

MT3: Intersection point of the fifth intercostal space with the left medio-clavicular line.

A, P1, MZ1, and MT3 are the classical auscultation sites which are called aortic, pulmonary, mesocardiac, and mitral auscultation sites, respectively.

In Fig. 7, second heart sounds recorded at 12 different sites for the third patient are given. These signals are high-pass filtered signals using the modal filtration technique described in Section II-B2). It can be seen that the temporal variation of these signals is different from site-to-site.

These sounds are then analyzed as explained above. For each recording site, model parameters are estimated. For 10 patients, parameters of modes with significant energies (more than $10 \%$ normalized energy) are given in Table V. It is possible to classify the modes, with respect to the frequencies, into different groups. For this purpose, the Average Linkage Method is used [18]. This method uses the Euclidean distance between data (or data groups). In each step, the distances of all possible pairs of data (or data groups) are calculated. The pair of data (or data groups) with the smallest distance constitutes a new group and the number of groups is decreased by one. The algorithm stops when the number of groups is decreased to the number initially given. Using this method in each patient, 4-5 groups are identified with different means. Then, means and coefficient of variations (ratio of the standard deviation to the group mean) of each group for all patients are computed (Table VI). In this table, the coefficient of variations do not exceed $5 \%$ in any of the mode groups.

It can be observed in Table $V$ that in each patient energies of modes change from site-to-site. In order to compare energies of modes between different sites, for each patient the ratios $E_{2} / E_{1}, E_{3} / E_{1}$, and $E_{4} / E_{1}$ are calculated for each recording site. It can be seen from Table $\mathrm{V}$ that, for several recording sites, Mode 1 (modes with the frequency of about $110 \mathrm{~Hz}$ ) does not exist. So, energy of this mode can be accepted as zero. However, for computation of the above-given energy ratios, assigning zero to $E_{1}$ yields infinity and makes statistical comparison impossible. For this reason, it is necessary to assign a value to the denominators of the energy ratios which are zero, so that statistical comparison is possible. For this 
TABLE $\mathrm{V}$

(a) Estimated Parameters of Patient 1. (b) Estimated Parameters of Patient 2
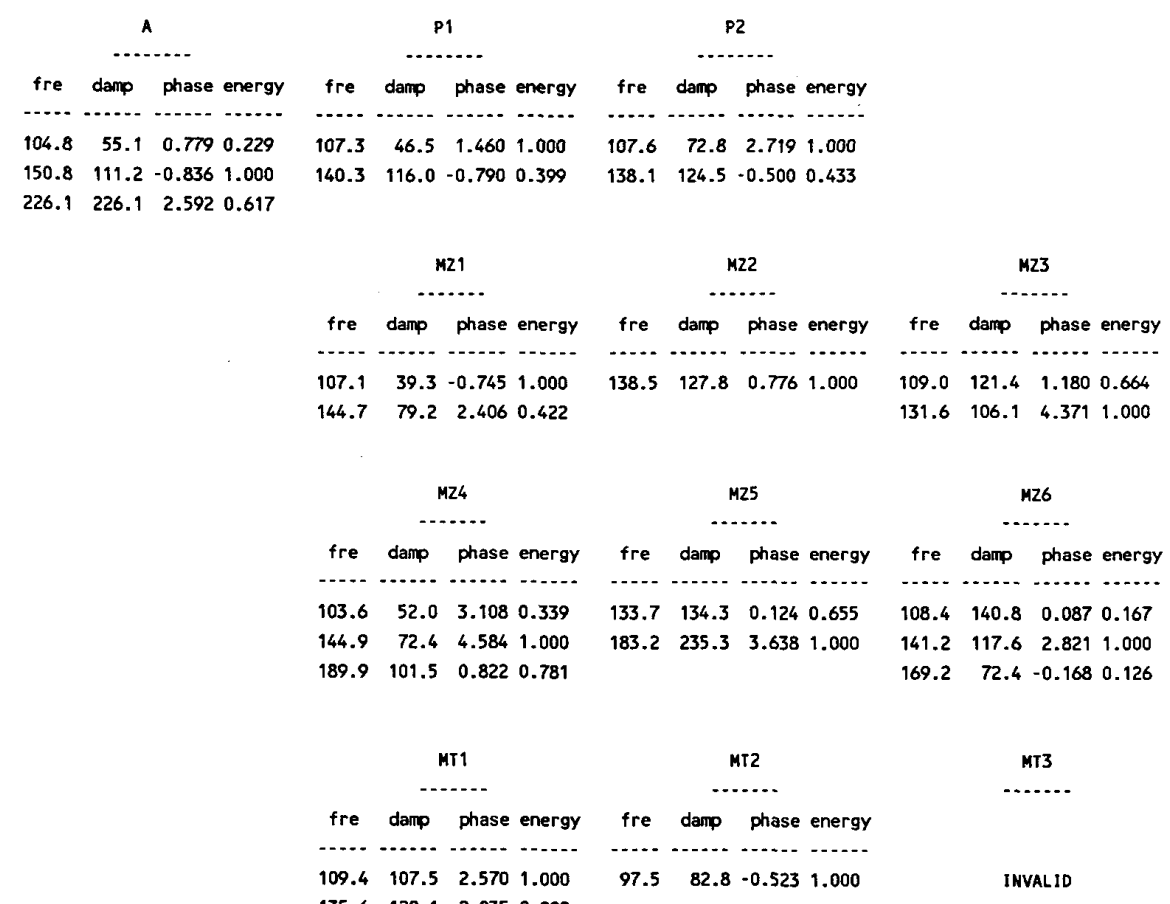

$\begin{array}{llll}135.6 & 128.1 & 2.835 & 0.892\end{array}$

(a)

\begin{tabular}{|c|c|c|c|c|c|c|c|c|}
\hline \multirow{3}{*}{ A } & \multicolumn{4}{|c|}{ P1 } & \multicolumn{4}{|c|}{ P2 } \\
\hline & & & $\cdots$ & & & & 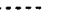 & \\
\hline & fre & damp & phase & energy & fre & damp & phase & energy \\
\hline \multirow{4}{*}{ INVAL ID } & -.... & ....... & 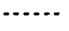 & 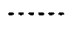 & $\cdots$ & ......... & 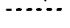 & 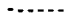 \\
\hline & 108.1 & 41.3 & 0.893 & 1.000 & 104.5 & 99.8 & 3.622 & 1.000 \\
\hline & 135.1 & 60.4 & 1.604 & 0.810 & 175.1 & 98.8 & -0.184 & 0.532 \\
\hline & 177.7 & 126.6 & 3.914 & 0.716 & & & & \\
\hline
\end{tabular}

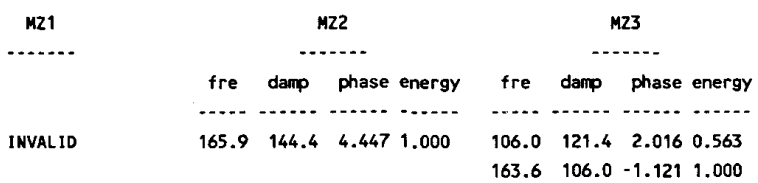

MZ4

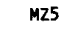

MZ6

fre damp phase energy fre............................

$123.3 \quad 87.3 \quad 0.544 \quad 1.000$ $\begin{array}{llll}161.4 & 143.4 & 2.488 & 0.650\end{array}$

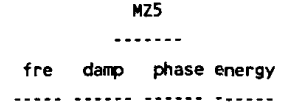

$\begin{array}{lrrr}105.7 & 75.3 & 0.435 & 1.000\end{array}$

$\begin{array}{lllll}175.6 & 106.2 & 1.421 & 0.826\end{array}$

$\begin{array}{lllll}211.1 & 168.8 & 3.399 & 0.292\end{array}$

fre damp phase energy

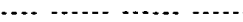

$\begin{array}{llll}162.9 & 94.8 & 1.647 & 1.000\end{array}$ $195.0 \quad 192.8-1.562 \quad 0.300$

MT1

\begin{tabular}{cccc}
\multicolumn{4}{c}{ MT2 } \\
\multicolumn{3}{c}{$\cdots$} \\
fre & damp & phase energy \\
\hline 104.5 & 49.9 & 1.217 & 1.000 \\
144.3 & 29.5 & 0.664 & 0.296
\end{tabular}

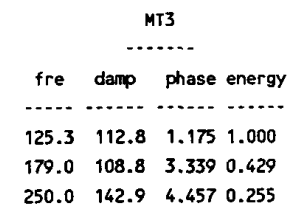

(b) 
TABLE V (Cont.)

(c) Estimated Parameters of Patient 3. (d) Estimated Parameters of Patient 4

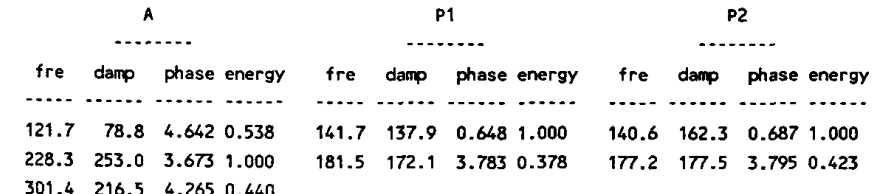

MZ1

fre damp phase energy

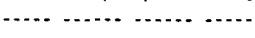

$134.8 \quad 99.6-1.177 \quad 0.260$

$175.7 \quad 242.3 \quad 1.490 \quad 1.000$

$229.9 \quad 183.6 \quad 3.097 \quad 0.199$

MZ4

fre damp phase energy (1.............................

$\begin{array}{llll}182.9 & 148.2 & 1.356 & 0.337\end{array}$ $229.6 \quad 221.6 \quad 3.905 \quad 1.000$

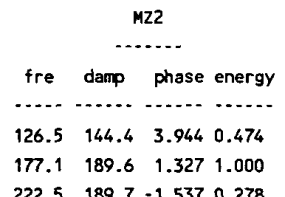

$222.5 \quad 189.7-1.5370 .278$

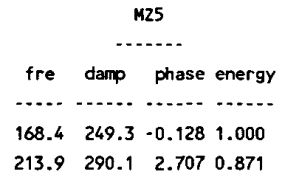

MT1

MT2

fre damp phase energy

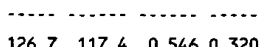

$186.0 \quad 128.0 \quad 4.019 \quad 0.865$

$234.2 \quad 147.4-0.083 \quad 1.000$

(c)

fre damp phase energy

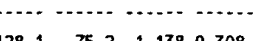
$168.9 \quad 186.2 \quad 4.310 \quad 1.000$

A

fre damp phase energy ................ $\begin{array}{lllll}110.6 & 102.0 & -0.427 & 0.626\end{array}$ $\begin{array}{lllll}170.9 & 145.6 & 1.483 & 1.000\end{array}$

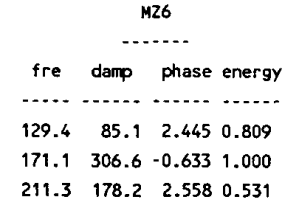

MT3

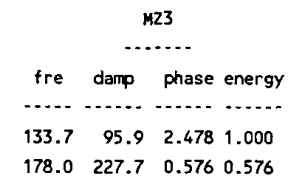

fre damp phase energy $139.3 \quad 101.0 \quad 3.427 .1 .000$ $\begin{array}{lllll}172.1 & 123.1 & 0.267 & 0.318\end{array}$

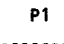

INVALID

\section{M21}

fre damp phase energy fre... -.... .............

$\begin{array}{llll}119.2 & 106.2 & 1.146 & 0.169\end{array}$ $\begin{array}{lllll}185.2 & 173.7 & 4.410 & 1.000\end{array}$ $\begin{array}{llll}232.0 & 250.5 & 0.193 & 0.457\end{array}$

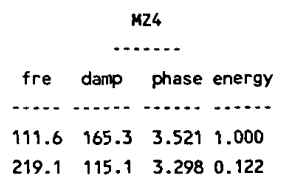

MT1

fre damp phase energy A.... -............. -......

$\begin{array}{lllll}109.8 & 70.8 & 3.593 & 0.230\end{array}$

$\begin{array}{lllll}155.6 & 96.3 & -0.993 & 1.000\end{array}$

$\begin{array}{lllll}225.4 & 171.2 & -0.542 & 0.926\end{array}$

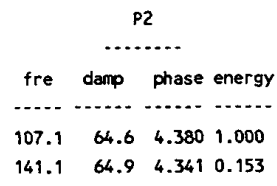

M22

fre damp phase energy ..... ....... -...........

$\begin{array}{llll}144.1 & 140.9 & 1.722 & 0.174\end{array}$ $207.3 \quad 132.8 \quad 3.866 \quad 1.000$ $251.7 \quad 230.0 \cdot 1.098 \quad 0.809$

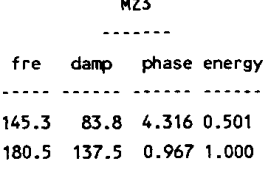

MZ6

fre damp phase energy fre.................... $\begin{array}{llll}131.0 & 130.7 \quad 3.603 & 1.000\end{array}$ -........$\begin{array}{llll}107.3 & 85.7 & 2.843 & 1.000\end{array}$ $\begin{array}{lllll}150.7 & 142.7 & -0.818 & 0.124\end{array}$ $249.3 \quad 235.2-1.3970 .114$

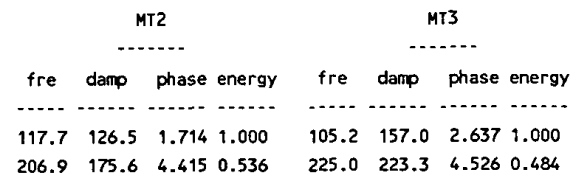

d) 
TABLE V (Cont.)

(e) Estimated Parameters of Patient 5. (f) Estimated Parameters of Patient 6
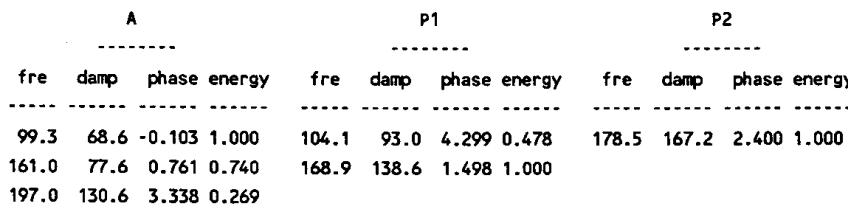
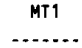

fre damp phase energy

..... ....... ..........

$\begin{array}{lllll}159.0 & 85.9 & 3.485 & 1.000\end{array}$ $\begin{array}{lllll}206.0 & 174.5 & 0.655 & 0.639\end{array}$

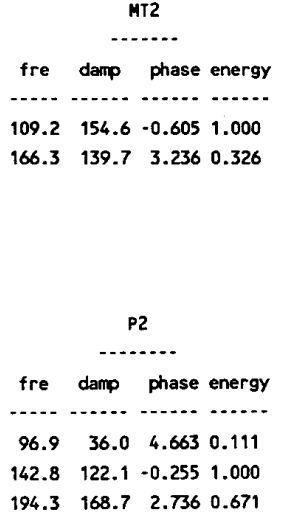
$\begin{array}{llll}142.8 & 122.1 & -0.255 & 1.000 \\ 194.3 & 168.7 & 2.736 & 0.671\end{array}$

(e)
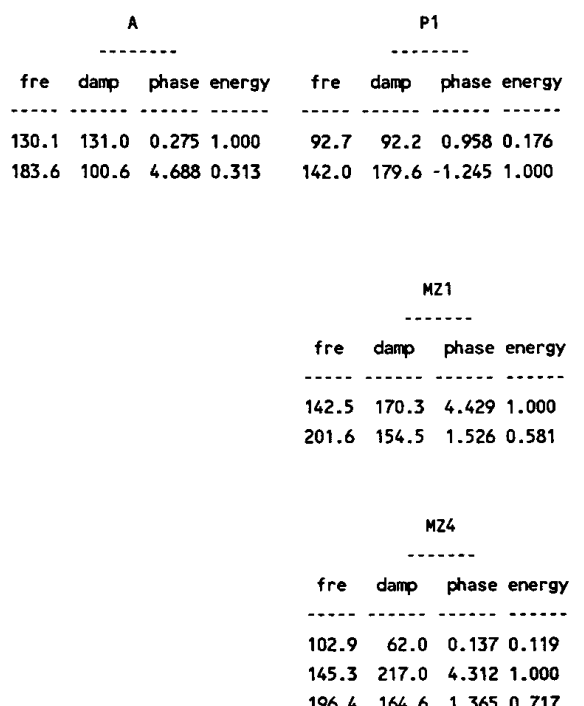

\begin{tabular}{cccc}
\multicolumn{4}{c}{$M 25$} \\
\multicolumn{4}{c}{$+\ldots \ldots$} \\
fre & damp & phase energy \\
\hline 106.0 & 130.0 & 4.207 & 1.000 \\
138.8 & 102.9 & 0.597 & 0.174
\end{tabular}

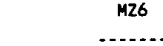

fre damp phase energy ..... -............. ...... $97.7 \quad 139.2 \quad 2.344 \quad 1.000$ $\begin{array}{llll}132.5 & 159.3 & -0.248 & 0.740\end{array}$

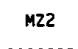

INVALID fre damp phase energy $99.0 \quad 136.2 \quad 3.314 \quad 1.000$ $\begin{array}{llll}138.4 & 119.6 & 0.983 & 0.573\end{array}$

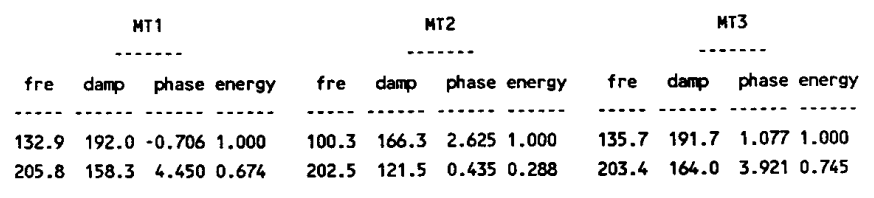

(f) 
TABLE V (Cont.)

(g) Estimated Parameters of Patient 7. (h) Estimated Parameters of Patient 8

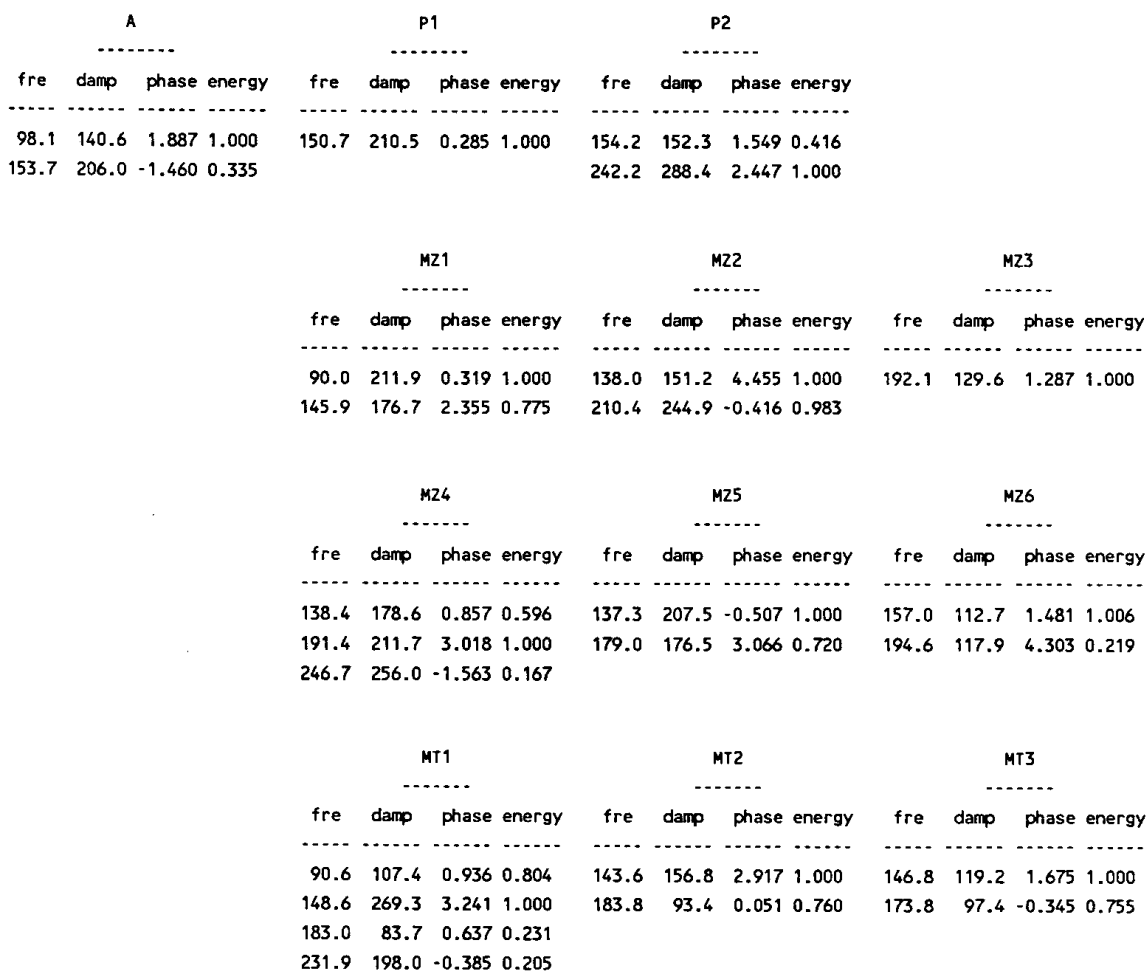

(g)

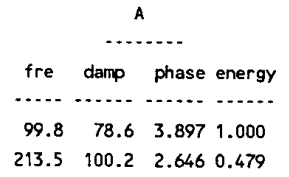

$\begin{array}{llll}213.5 & 100.2 & 2.646 & 0.479\end{array}$
P2

fre damp phase energy

................... .....

$\begin{array}{llll}133.0 & 42.6 \quad 4.426 & 0.210\end{array}$ $187.9 \quad 107.4 \quad-0.956 \quad 1.000$

$\begin{array}{lllll}134.9 & 57.7 & 3.793 & 0.169\end{array}$

$205.7 \quad 112.2 \quad 3.762 \quad 1.000$

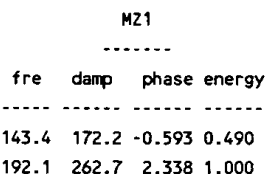

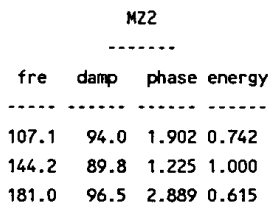

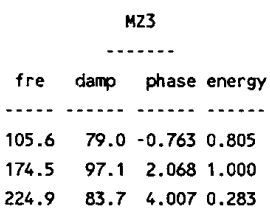

\begin{tabular}{cccc}
\multicolumn{4}{c}{$M Z 4$} \\
fre & damp & phase energy \\
\hdashline 108.8 & 86.8 & 1.824 & 1.000 \\
132.1 & 84.8 & -0.287 & 0.507
\end{tabular}

\begin{tabular}{|c|c|c|c|}
\multicolumn{4}{c}{ MZ5 } \\
\multicolumn{4}{c}{$\ldots$} \\
fre & damp & \multicolumn{1}{c}{ phase energy } \\
\hline 142.1 & 101.6 & 0.806 & 1.000 \\
\hline 184.9 & 93.0 & 3.699 & 0.239 \\
\hline
\end{tabular}

\begin{tabular}{crrr}
\multicolumn{4}{c}{$M 26$} \\
\multicolumn{4}{c}{$\ldots$} \\
fre & damp & phase energy \\
\hline$\ldots . .$. & $\ldots . .$. & $\ldots . .$. \\
\hline 110.8 & 76.7 & 3.903 & 1.000 \\
149.8 & 120.8 & 0.446 & 0.384
\end{tabular}

\begin{tabular}{cccc}
\multicolumn{4}{c}{ MT1 } \\
\multicolumn{4}{c}{$\cdots \ldots .}$. \\
fre & damp & phase energy \\
\hdashline 101.3 & 49.5 & 0.706 & 1.000 \\
132.1 & 39.5 & 3.060 & 0.178 \\
198.4 & 47.0 & -0.793 & 0.137
\end{tabular}

MT2

fre damp phase energy

$\begin{array}{lllll}110.1 & 183.4 & 3.728 & 0.758\end{array}$

$\begin{array}{lllll}140.4 & 110.8 & 0.548 & 1.000\end{array}$

fre damp phase energy

$104.5 \quad 120.3 \quad 4.616 \quad 0.745$

$\begin{array}{lllll}143.7 & 170.0 & 0.942 & 1.000\end{array}$ 
TABLE V (Cont.)

(i) Estimated Parameters of Patient 9. (j) Estimated Parameters of Patient 10
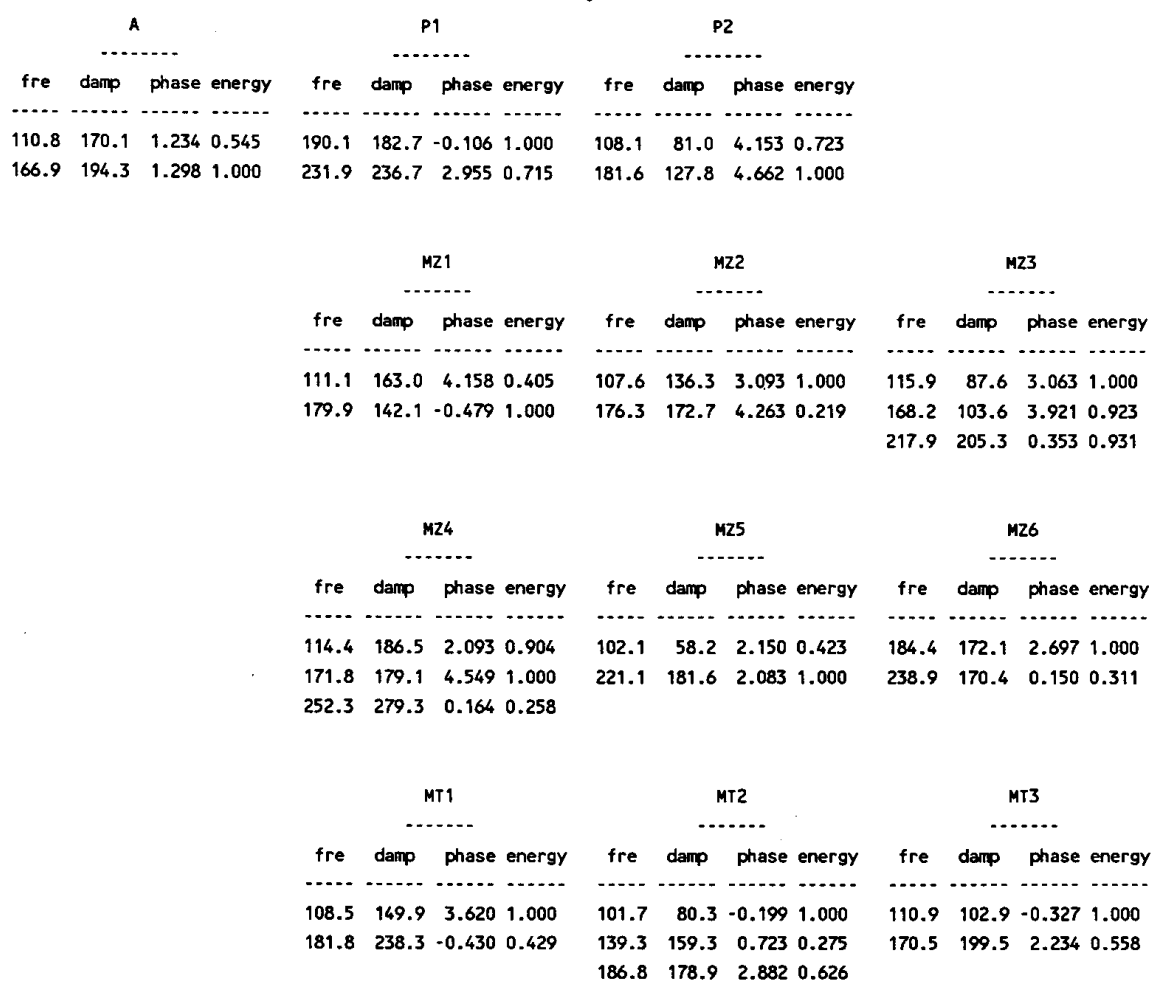

(i)
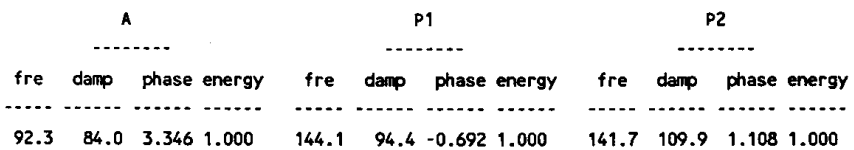

MZ1

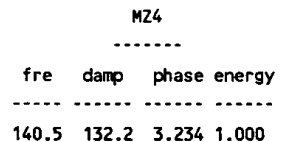

$\begin{array}{llll}140.5 & 132.2 \quad 3.234 & 1.000\end{array}$

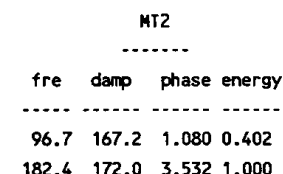

$182.4 \quad 172.0 \quad 3.532$

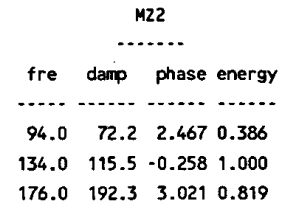

M25

fre damp phase energy f................... -....

$132.3 \quad 190.1-1.330 \quad 1.000$ $\begin{array}{llllll}223.9 & 238.0 & 2.833 & 0.606\end{array}$

) 
TABLE VI

Means (Hz) and Coefficient of Variations (in Parentheses, \%) of Modal Frequencies for the Patients. The Groups for Which Coefficient of Variations Are Not Given Consist of Only One Element

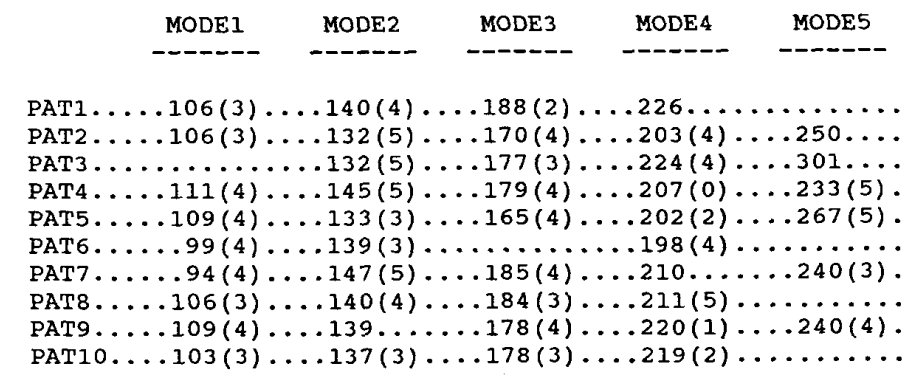

purpose, a value of 20 is assigned to the above-mentioned energy ratios. It should be emphasized that statistical analyses results do not change when this value is chosen between $10-100$

In Table VII, means and standard deviations of energy ratios are given for 12 different recording sites. It can be observed from this table that means of energy ratios vary from site-tosite. To observe if the variation of energy ratios on the surface of the chest is statistically significant, we first used the one-way analysis of variance test. As a result of this test, no significant difference is observed (Table VIII). The analysis of variance test gives information about the variation of overall data and a significant difference between several pairs of groups may be suppressed. According to the resonant structure hypothesis, energies of modes will vary depending on the distance of recording sites to the related anatomical structures. However, it does not imply that energy ratios will be different in each recording site. In fact, according to this hypothesis, energy ratios may be the same at adjacent recording sites which are close to the same part of the heart. As a result of the analysis of variance test, similarity of energy ratios at adjacent recording sites may suppress the significant difference between several recording sites. Therefore, energy ratios are also analyzed by using a $t$-test to observe if there are significantly different pairs of recording sites. The significance level for $t$-tests is chosen as $p<0.01$. As a result, $E_{2} / E_{1}$ and $E_{4} / E_{1}$ are found to be significantly higher at MZ2 with respect to MT2 $(p<0.01)$. When the significance level for the $t$-test is accepted as $p<$ 0.05 , additional significantly different pairs of recording sites are observed. In Table IX, these recording sites are given. It can be observed from this table that the energy ratios $E_{2} / E_{1}$ and $E_{4} / E_{1}$ are found to be significantly higher for MZ2 with respect to several other recording sites.

\section{CONCLUSION}

Heart sound signal as recorded on the surface of the chest is related to its generation mechanism in a very complex manner. The work presented in this paper describes a methodology for identifying a model which emerges from the hypothesis that a closure sound of a mechanical prosthesis is the output a linear system of acoustic resonating structures excited by a short pulse generated during rapid deceleration of valve occluder [12]. This acoustic system, made up of the heart,
TABLE VII

Mean and Standard Deviation of Energy Ratios Obtained for DifFerent Recording Sites

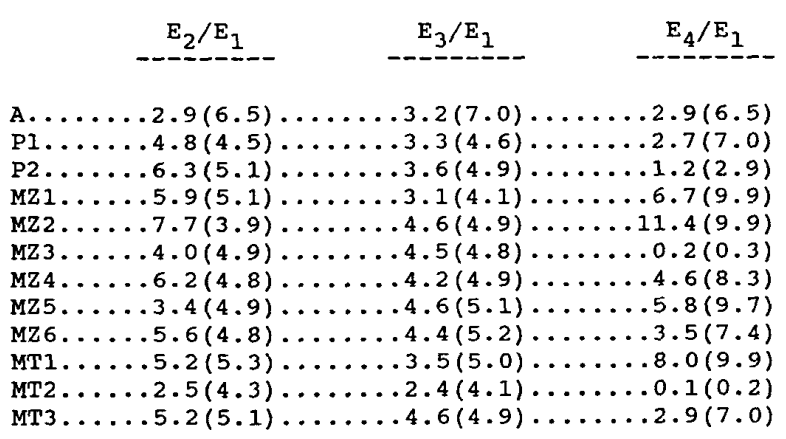

TABLE VIII

$p$ Values as a Result of the One-Way ANalysis OF VARIaNCE TEST FOR THE ENERGy Ratios

\begin{tabular}{ccc}
$\mathrm{E}_{2} / \mathrm{E}_{1}$ & $\mathrm{E}_{3} / \mathrm{E}_{1}$ & $\mathrm{E}_{4} / \mathrm{E}_{1}$ \\
\hline 0.29 & 0.47 & 0.19
\end{tabular}

TABLE IX

In Each Pair, Energy Ratio of the First Recording Site Is Found to Be Significantly Higher WITH RESPECT to the Second Recording Site $(p<0.05)$. The Pairs Which Are SignificANTLY DifFERENT fOR $p<0.01$ ARE MARKed By $\left({ }^{*}\right)$

\begin{tabular}{lll}
$\mathrm{E}_{2} / \mathrm{E}_{1}$ & $\mathrm{E}_{3} / \mathrm{E}_{1}$ & $\mathrm{E}_{4} / \mathrm{E}_{1}$ \\
\hdashline $\mathrm{MZ2}-\mathrm{A}$ & - & $\mathrm{MZ2}-\mathrm{A}$ \\
$\mathrm{MZ2}-\mathrm{MZ3}$ & - & $\mathrm{MZ2}-\mathrm{P1}$ \\
$\mathrm{MZ2}-\mathrm{MZ5}$ & $\mathrm{MZ2}-\mathrm{P2}$ \\
$\mathrm{MZ2}-\mathrm{MT2}{ }^{*}$ & & $\mathrm{MZ2}-\mathrm{MZ3}$ \\
& & $\mathrm{MZ2}-\mathrm{MZ} 6$ \\
& $\mathrm{MZ2}-\mathrm{MT2}$ \\
& $\mathrm{MZ2}-\mathrm{MT3}$ \\
& $\mathrm{MT1}-\mathrm{MZ3}$ \\
& $\mathrm{MT1}-\mathrm{MT2}$ \\
& $\mathrm{MZ1-MT2}$
\end{tabular}

its partitions, and major vessels, is a highly complex system containing many coupled elements. The task of identification is further complicated by noise and measurement problems. These problems have been addressed in this paper on a quantitative basis, both by simulation and by analysis of real signals. It is demonstrated that the number of modes necessary 
to model the system varies with noise, even after the Akaike Criterion is met; the identification of mode parameters is highly dependent on noise level; and for correct estimation of parameters of modes of interest, it is necessary to employ a sophisticated technique to reduce the effect of the lowfrequency-high-energy modes.

It is observed that the coefficient of variation of modal frequencies in any of the patients does not exceed $5 \%$ over the surface of the chest when the methodology presented in this paper, i.e., signal averaging, modal high-pass filtering and the given order selection method, is employed. This variation is within the accuracy limits of the method. Therefore, the hypothesis of resonant acoustic structures is strongly supported by this result. Furthermore, in line with the implications of the same hypothesis, it is found that relative energies of resonance modes vary across the chest surface. Although a consistent pattern of variation is not found, statistical analyses have shown that two sites are significantly different with respect to their energy ratios $E_{2} / E_{1}$ and $E_{4} / E_{1}(p<0.01, N=10)$.

Application of the proposed method to different patient populations and correlation of mode parameters to the position and physical dimensions of the internal structures can be helpful in associating a particular mode to a particular structural element. For example, patients with left ventricular dilatation or other cases where the volumes of different partitions in the heart or vessels are known to be altered should have shifted mode frequencies compared to normals. The study of such patients will help establish the relation between modal parameters and the structures.

\section{ACKNOWLEDGMENT}

The authors wish to thank Prof. S. Yücemen for valuable advice during the statistical analysis of data.

\section{REFERENCES}

[1] J. C. Hylen, F. E. Kloster, R. H. Herr, A. Starr, and H. E. Griswold "Sound spectrographic diagnosis of aortic ball variance," Circ., vol. 39, pp. 849-858, June 1969.

[2] R. F. Gordon, M. Najimi, B. Kingsley, B. L. Segal, and J. W. Linhart, "Spectroanalytic evaluation of aortic prosthetic valves," Chest, vol. 66, pp. 44-49, July 1974.

[3] Y. Kagawa, S. Nitta, M. Tanaka, and T. Horiouchi, "Real-time sound spectroanalysis for diagnosis of malfunctioning prosthetic valves," $J$. Thorac. Cardiovasc. Surg., vol. 79, pp. 671-679, 1980.

[4] P. D. Stein, H. N. Sabbah, J. B. Lakier, and D. J. Magilligan, "Frequency of the first heart sound in the assessment of the stiffening of mitral bioprosthetic valves," Circ., vol. 63, pp. 200-203, Jan. 1981.

[5] P. D. Stein, H. N. Sabbah, J. B. Lakier, and S. R. Kemp, "Frequency spectra of the first heart sound and of the aortic component of the second heart sound in patients with degenerated porcine bioprosthetic valves," Amer. J. Cardiol., vol. 53, pp. 557-561, Feb. 1984.

[6] L. G. Durand, M. B. Blanchard, G. Cloutier, H. N. Sabbah, and P. D. Stein, "Comparison of pattern recognition methods for computerassisted classification of spectra of heart sounds in patients with a porcine bioprosthetic valve implanted in the mitral position," IEEE Trans. Biomed. Eng., vol. BME-37, pp. 1121-1129, Dec. 1987.

[7] R. J. Adolph, J. F. Stephens, and K. Tanaka, "The clinical value of frequency analysis of the first heart sound in myocardial infarction," Circ., vol. 41, pp. 1003-1014, June 1970.

[8] W. B. Clarke, S. M. Austin, P. M. Shah, and P. M. Griffen, "Spectral energy of the first heart sound in acute myocardial ischemia," Circ., vol. 57, pp. 593-598, Mar. 1978.

[9] J. Semmlow, W. Welkowitz, J. Kostis, and J. W. Mackenzie, "Coronary artery disease-Correlates between diastolic auditory characteristic and coronary artery stenoses," IEEE Trans. Biomed. Eng., vol. BME-30, pp. 136-139, Feb. 1983.

[10] P. J. Amott, G. W. Pfeiffer, and M. E. Tavel, "Spectral analysis of heart sounds: Relationships between some physical characteristics and frequency spectra of first and second heart sounds in normals and hypertensives," J. Biomed. Eng., vol. 6, pp. 121-128, Apr. 1984.

[11] M. Akay, J. L. Semmlow, W. Welkowitz, M. D. Bauer, and J. B. Kostis, "Detection of coronary occlusions using autoregressive modeling of diastolic heart sounds," IEEE Trans. Biomed. Eng., vol. 37, pp. 366-373, Apr. 1990.

[12] H. Köymen, B. K. Altay, and Y. Z. İder, "A study of prosthetic heart valve sounds," IEEE Trans. Biomed. Eng., vol. BME-34, pp. 853-863, Nov. 1987.

[13] D. L. Sikarskie, P. D. Stein, and M. Vable, "A mathematical model for aortic valve vibration," J. Biomechan., vol. 17, pp. 831-837, 1984.

[14] F. Meno, P. S. Reddy, and L. Bemardi, "Heart sound propagation in the human thorax" Clin. Phys. Physiol. Meas., vol. 6, pp. 123-129, 1985.

[15] S. M. Kay and L. Marple, "Spectrum analysis-A modern perspective," Proc. IEEE, vol. 69, pp. 1380-1419, Nov. 1981.

[16] H. Akaike, "A new look at the statistical model identification," IEEE Trans. Automat. Contr., vol. AC-19, pp. 716-723, Dec. 1974.

[17] M. E. Tavel, Clinical Phonocardiography and External Pulse Recording. Chicago: Year Book Med. Publ., 1985, p. 15.

[18] R. A. Johnson and D. W. Wichern, Applied Multivariate Statistical Analysis. Englewood Cliffs, NJ: Prentice-Hall, 1982, p. 552.

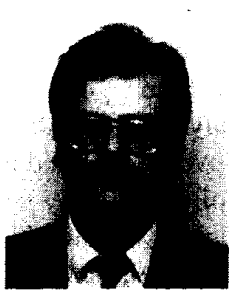

Ahmet Baykal was bom in K. Maras, Turkey on September 27, 1961. He received the M.D. degree from Aegean University in 1984 and the Ph.D. degree in biomedical engineering from Middle East Technical University in 1992. He did postdoctoral studies at the Biomedical Engineering Department of The Johns Hopkins University between 1993 and 1994.

His main research areas are biomedical signal processing, modeling and analysis of phonocardiogram, and electrophysiology of ventricular fibrillation and cardio-pulmonary resuscitation.

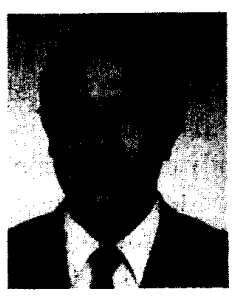

Y. Ziya İder was bom in Erzincan, Turkey, on October 9, 1951. He received the B.Sc. degree in electrical engineering from Middle East Technical University in 1973, the M.Sc. degree in biomedical engineering from the University of Southem California in 1976, and the Ph.D. degree in biomedical engineering from Northwestern University in 1979. In 1979, he joined the Department of Electrical and Electronics Engineering of Middle East Technical University, where he is currently employed as a Professor. His main research areas are acquisition and processing of bioelectric and other physiological signals, electrical impedance tomography, and magnetic resonance imaging.

Hayrettin Köymen (S'74-M'87-SM'91) was born in Ankara, Turkey, on June 7, 1952. He received B.Sc. and M.Sc. degrees from Middle East Technical University, Ankara, Turkey, in 1973 and 1976, respectively, and the Ph.D. degree from the University of Birmingham, England, in 1979, all in electrical engineering.

In 1979, he became a faculty member of the Middle East Technical University. Until 1982, his work involved underwater acoustics and oceanographic instrumentation. Since 1982, his work has involved physiological signal acquisition and processing, medical ultrasonics, and ultrasonic nondestructive evaluation. In 1990, he joined the faculty of Bilkent University, Ankara, where he is now a Professor in the Department of Electrical and Electronics Engineering. His current research interests are personal computerbased biomedical signal acquisition and processing, medical imaging, finite amplitude effects in medical ultrasonics, and acoustic microscopy using leaky waves in layered media. 